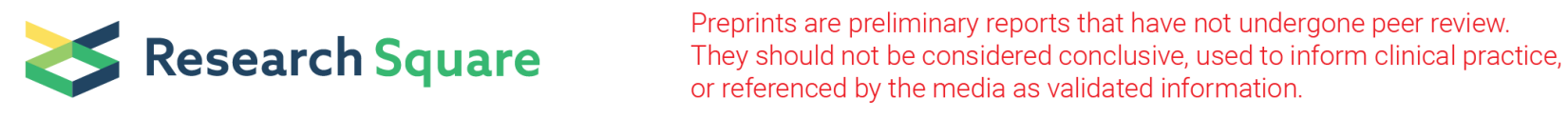

\title{
Correlation of Synovial Tissues Protein Abundance With Age and Menopause in Osteoarthritis and Knee Joint Injury of Female Patients
}

\author{
Xiao Yang \\ Tianjin Medical University \\ Zhe Fu \\ Tianjin Medical University \\ qiuyu ren \\ Chengdu Medical College https://orcid.org/0000-0002-4480-3695 \\ Shuang Wang \\ Sichuan Academy of Medical Sciences and Sichuan People's Hospital \\ Shuai Yin \\ Tianjin Medical University \\ Jun Liu ( $\sigma_{\text {tjliujun@tmu.edu.cn ) }}$ \\ Tianjin Medical University https://orcid.org/0000-0002-5391-0003
}

\section{Research Article}

Keywords: Proteome, Osteoarthritis, Synovial tissues, age, menopause women

Posted Date: May 27th, 2021

DOI: https://doi.org/10.21203/rs.3.rs-535550/v1

License: (1) This work is licensed under a Creative Commons Attribution 4.0 International License. Read Full License 


\section{Abstract}

\section{Background}

Knee injury and osteoarthritis (OA) are common articular disorders that affect numerous people of different ages, particularly menopausal women. Epidemiological surveys conclude that ladies gain a higher prevalence of OA than men, the menopausal women even get higher morbidity. However, studies about OA animal models suggest that the animals could remain a good joint function regardless of aging and menopause; age and menopause seem to have little effect on joint function. Thus, the study aimed to explore whether age and menopause will influence the clinical recovery of a knee joint in $\mathrm{OA}$ and injury women and the relevant mechanism by analyzing the proteome profile of synovial tissue (ST) samples.

\section{Methods}

The routine blood test and hormone levels were detected before the surgery. The ST samples of participants were collected during the surgery and 8 ST samples were used for proteome analysis. Just the day before and one month after the surgery, scores of Visual Analogue Score (VAS), Lysholm, The Western Ontario and McMaster Universities Osteoarthritis Index (WOMAC) were used for assessing the functions of the affected knee. The relationship among proteome data, aging, estrogen level, and affected knee function were analyzed.

\section{Results}

Age, menopause and disorders of the knee were related to the clinical outcomes of knee OA and injuries of female patients. Proteome data revealed that the abundance of 174 proteins in old women was statistically different from those of young women. Similarly, compared to the menopausal women, about 120 proteins were significantly changed in the samples taken from premenopausal women. 58 proteins were shown to be insignificant different amounts in ST from knee OA women compared to knee injury ones. The reactions and pathways of these proteins were analyzed and visualized.

\section{Conclusions}

Age and menopause showed a certain correlation with the protein profile of ST from OA or knee injury female patients, which indicated that the protein components might be affected by age and menopause. Besides, age and menopause also influenced the clinical outcomes of these patients after surgery therapy. Aging and menopause might take part in the regulation of remaining knee joint functions via modeling the ST protein expression.

\section{Background}

Osteoarthritis $(\mathrm{OA})$ is a common complaint in young and old people, causing the patients many inconveniences in daily life and work. Therefore, epidemiological studies and controlled trials are carried out by many researchers. Based on the previous surveys, age, estrogen, sex, dietary intake et al are important risk factors for the initiation and development of $O A[1,2,3,4,5]$. Besides, menopause is also suggested to be associated with the onset and progression of OA in women [6]. The involvement of all of the above risk factors in the progression of osteoarthritis requires further research.

Synovial tissues (ST) have also been shown to be involved in remaining the joint function and the incidences of many joint diseases[7]. Essential components expressed in ST are of great significance in regulating the functions of the knee joint and the responses to multifarious joint disorders. For example, the prolactin receptor (PRLR), expressed by macrophages, is detected in ST samples from both OA and rheumatoid arthritis (RA) patients, stimulating the activation of macrophages with other pro-inflammatory cytokines [8,9,10]. $\beta 1,4-g a l a c t o s y l t r a n s f e r a s e-I$ $(\beta 1,4-\mathrm{GalT}-\mathrm{I})$ was also reported to be elevated in ST of OA patients [11]. However, many other proteins that might also contribute to the incidence and the development of OA in ST are not fully identified. Therefore, in the study, we purpose to explore the association of aging, menopause and OA by characterized ST proteomic expression patterns together with clinical outcomes.

\section{Methods}

\section{Participants}

Synovial tissue samples were obtained from female patients who suffered from knee OA or knee injury and underwent surgical therapy from Nov. of 2018 to April of 2019 in Sichuan Provincial People's Hospital. To be specific, patients who fitted the following criterion were included: (1) female patients with knee OA or knee injury, (2) aged at 20 70y, (3) without other maladies of bone and articular, or any pre-therapy history of the knee OA or knee injury, (4) signed the study consent and was willing to be follow-up. Although 21 patients were included, only samples from 8 patients were applied for proteomic analysis regarding their comparability. 
The peripheral blood used for estrogen testing was obtained on the morning of the operative day by nurses and was sent to the Hospital's testing center immediately.

\section{Protein preparation and digestion}

The synovial tissue (ST) samples were obtained during the surgery and immediately stored in liquid nitrogen. Protein extraction was performed according to the requirements of MS analysis. Briefly, $20 \mathrm{mg}$ of ST sample was ground and sonicated (Q800R sonicator, QSONICA, Newton, Connecticut, USA) in $1 \mathrm{ml}$ lysis buffer (7M urea, $4 \%$ SDS, $1 \mathrm{mM}$ phenylmethane sulfonyl fluoride (PMSF), 2mM EDTA, 10mM dithiothreitol (DTT), $1 \times$ protease inhibitor cocktail (Sigma-Aldrich, St. Louis, USA) in 30mM HEPES, pH 7.4). The insoluble content in the lysate was discarded by centrifugation at $13000 \mathrm{~g}$ and $4{ }^{\circ} \mathrm{C}$ for $10 \mathrm{~min}$. The protein concentration of the supernatant was determined with a BCA Protein Assay Kit (Thermo Fisher Scientific, Bonn, Germany) and the quality of the samples was evaluated via the SDS-PAGE method. The samples were then precipitated with $15 \%$ trichloroacetic acid for $2 \mathrm{~h}$ at $4{ }^{\circ} \mathrm{C}$ and washed with acetone. The precipitates were redissolved in triethylammonium bicarbonate buffer. The solutions were then digested with trypsin (Promega, Madison, WI, USA) at an enzyme/ substrate ratio of $1: 50$ at $37^{\circ} \mathrm{C}$ for $16 \mathrm{~h}$

\section{TMT labeling, HPLC fractionation}

The digested peptide mixtures were first desalted using a Sep-Pak C18 column (100mg, 1 cm³; Waters Corporation, Milford, MA, USA). TMT labeling was performed according to the manufacturer's instructions. Briefly, anhydrous acetonitrile was mixed with TMT Reagents preequilibrated to room temperature. The mixture was added to each sample, and the purified samples were chemically labeled with the

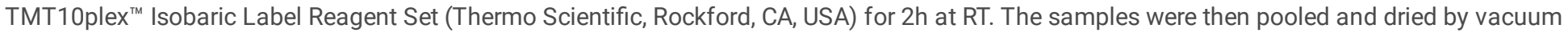
centrifugation. The dried samples were acidified with $0.1 \%$ trifluoroacetic acid and desalted using Sep-Pak C18 $\left(100 \mathrm{mg}, 1 \mathrm{~cm}{ }^{3} ;\right.$ Waters Corporation, Milford, MA, USA) and fractionated through a BEH C18 column $(2.1 \times 150 \mathrm{~mm}, 1.7 \mu \mathrm{m}, 130 \AA ̊)$ using ACQUITY UPLC $\AA$ H-Class System (Waters Corporation, Eschborn, Germany) at a flow rate of $250 \mu \mathrm{l} / \mathrm{min}$.

\section{LC-MS/MS analysis}

The eluted fractions were collected and combined into 12 fractions for further LC-MS/MS analysis. The settings of MS were as follows: ESI voltage, $2 \mathrm{kV}$; inlet capillary temperature, $300^{\circ} \mathrm{C}$; full-scan automatic gain control (AGC) target, $5 \times 10^{5}$ ions at 60,000 resolution; scan range, $400-$ $1600 \mathrm{~m} / \mathrm{z}$; Orbitrap full-scan maximum injection time, $50 \mathrm{~ms}$; MS/MS scan AGC target, $2 \times 10^{5}$ ions at 30,000 resolution; and maximum rejection, $150 \mathrm{~ms}$.

\section{Evaluation of clinical outcomes}

Just the day before and one month after the surgery, joint functions of patients were evaluated by scores of Visual Analogue Score (VAS), Lysholm, The Western Ontario and McMaster Universities Osteoarthritis Index (WOMAC). All the evaluations were conducted by the same group of doctors.

\section{The analysis of reactions and pathways among proteins}

The immune or endocrine system-related proteins which were insignificant abundances between old OA patients and young OA patients were selected out by the ClueGO tool of Cytoscape (v3.7.2) and were analyzed with Reactions and Pathways of Reactome program provided online (https://reactome.org/)

\section{Statistical analysis}

The proteomic raw data was initially processed with Proteome Discovery 1.4 (Thermo Fisher Scientific, Waltham, MA, USA). KOBAS 3.0 (http://kobas.cbi.pku.edu.cn/) [12], a web server for gene/protein functional annotation and functional gene set enrichment, was used for pathway and Gene Ontology functional category enrichment analysis. The false discovery rate (FDR) was calculated by the method described by Benjamin. Significant results were determined based on FDR-adjusted p-value $\leq 0.05$. SPSS version 17.0 (SPSS Inc., Chicago, IL) was used for the data analysis, and $P$ value $<0.05$ was considered as statistically significant. The data of BMI, Mean age, VAS, Lysholm, WOMAC scores were shown as the mean \pm standard deviation. The student's t-test was applied for comparing the means of two independent groups and one-way ANOVA analysis was used to compare the means among three groups. Pearson's correlation and linear regression analysis were used to evaluate the correlation between the concentration of estrogen and the VAS, Lysholm as well as WOMAC scores.

\section{Results}


The effects of aging and menopause on the clinical outcomes of knee $\mathrm{OA}$ and injury women

The basic information of the participants whose ST samples were analyzed as well as the estrogen levels, the pre-surgery and post-surgery evaluated scores were summarized in Table 1. Aging, menopause, or estrogen levels were essential factors that would result in a change of postsurgery function outcomes. Even though all participants got a better score after a month of operative therapy, young patients exhibited more agreeable outcomes compared to the aging ones regardless of menopause and disease types $(F D R<0.05)$; on the other hand, premenopausal ladies also enjoyed more satisfying function outcomes than menopausal ones ( $F D R<0.05)$. However, no differences in post-surgery function recovery were detected comparing women with higher levels of estrogen to women whose estrogen levels were much lower.

\section{Characteristics of the proteome profile of ST from knee OA and knee injury female patients}

Around 4000 proteins were detected in the proteome and finally, 3465 proteins were used for the analysis after quantifying (FDR<0.01). The proteins were composed of different cellular components and were involved in various biological processes (Fig.1), according to GO and KEGG analysis.

\section{Proteins profile in ST from OA and knee injury female patients}

Our results displayed that ST protein expression patterns were affected by age, menopause, and disease types which were grouped into knee OA group and knee injury group. The average counts of the top 20 candidate proteins which would be most affected by age, menopause, or disease types estrogen levels were listed in Table 2.

To better understand whether the protein profiles were affected by estrogen, the estrogen levels were also tested. The differently expressed proteins were as shown in $(F D R<0.01)$ Table 2. The relationship between estrogen levels and protein profiles was identified by Pearson correlation analysis. Yet, no significant correlation in protein expression patterns and estrogen levels was detected.

The protein abundances of ST were affected by aging. The protein profiles varied: some proteins were upregulated and the expression levels of some proteins decreased from ST in young female OA and knee injury patients while compared to aging participants (Fig.2a and b).

The protein profiles were also influenced by menopause. In comparisons of menopausal knee OA and knee injury patients to the premenopausal ones, some protein levels such as DCTN5, AKR7A2, and NHP2 were significantly increased, while proteins like HDGFL3, MYCBP2 and PRS15 were markedly decreased in ST samples (Fig.2c).

Interestingly, the protein profiles of ST from female knee OA patients also differed from those of knee injury women (Fig.2d). Female knee OA patients exhibited a higher level of proteins such as TRADD, CTSZ and LZIC; yet a lower level of proteins such as ZFYVE26, LRRC25 and QPRT compared with the knee injury ones.

\section{Network analysis of candidate proteins}

Candidate proteins that would be affected by aging, menopause, disease types and estrogen levels were further analyzed by network analysis. The protein-protein interaction network was displayed in Fig.3: candidate proteins that mostly affected by aging, as well as their interacted partners, were showed in Fig.3a, and then the candidate molecules which significantly influenced by menopause (Fig.3b), the ones that were correlated with disease types (Fig.3c) and estrogen levels (Fig.3d).

\section{The reactions and pathways analysis of candidate proteins analyzed by the Reactome program}

The ST proteome data were used for further analysis by the Reactome program. Because the development of OA is associated with the immune system and endocrine system, candidate proteins that were indifferent abundances in ST proteome between menopausal and premenopausal participants were analyzed and those related to the immune or endocrine system were selected. Then those selected proteins were analyzed by Reactions and Pathways in the Reactome program. The potential reactions and pathways among these proteins were shown in Fig.4, which might provide insight into the mechanism by which menopause contributes to the development of OA.

\section{Discussion}

$\mathrm{OA}$ is a leading cause of joint pain and disability, affecting a substantial proportion of people worldwide [13]. Therefore, more and more investigators focus on proteomics studies investigating the componence and potential roles of synovial fluid [14,15,16,17,18]. Animal models of osteoarthritis are also used in recent studies to understand the pathophysiology of OA and develop successful treatment of OA [19,20]. However, the role of ST of the knee joint in the onset and development of OA remains largely unknown. In this study, we explored the relationship between the recovery of OA after surgery, age, menopause and ST proteome data, aiming at providing insights for mechanisms of the onset and development of OA. 
As shown in the results above, aging is the key factor that may influence the post-surgery recovery of knee OA or injury patients, but menopause is not associated. No statistical differences were observed while in comparison to participants with different estrogen levels. The reason might be that estrogen levels may vary according to the period of studied women; the whole picture of their regular mean estrogen levels could not be represented by several tests. Namely, our data of the analyzed estrogen levels were not comprehensive. Since those data might not comprehensively reflect the actual estrogen levels, we were unable to figure out whether estrogen contributes to the remaining joint functions or not. Yet, the role of estrogen in OA initiation and development was inconclusive. According to some surveys, estrogen did contribute to the recovery of $O A$ and could markedly reduce the prevalence of $O A$ [21. In vivo data also suggested that estrogen played an anti-inflammation role in OA animal models [22]. By inhibiting the development of joint swelling and reducing the release of inflammatory cytokines, estrogen could effectively relieve much joint pain and improve the joint swelling situation [22]. Therefore, some estrogen drugs were applied for OA treatment. As one of the OA therapy strategies, estrogen drugs were evaluated by many investors, yet the results and conclusions were inconsistent among the clinical surveys $[21,23,24]$. Such a phenomenon might be a consequence of various causes.

We demonstrated that the protein profiles vary with aging, menopause, as well as disease types. The proteomic data revealed that the protein composition of old OA women differed from those of young OA women. Our data is consistent with the former report [3]. Unfortunately, perplexed by how age affected human life for centuries, scientists are still unable to explain the aging problem. According to the current technology, we cannot explain how aging leads to the difference in protein mass spectrum? We are also not clear whether such differences are a cause of aging or are a consequence of aging.

It is well established that menopause leads to a significant change of expression levels of some special genes. However, to our best knowledge, similar influences caused by estrogen levels in ST have not been well studied. According to our data, there were no remarkable differences in ST protein expression levels between patients with different estrogen levels at comparable ages, which is quite adverse to the results of a significant association between ST protein profile and menopause. This paradox may be the result of the dynamic fluctuation of estrogen levels in premenopausal women. Women with perimenopausal estrogen levels vary greatly, estrogen levels can be very high.

Similarly, it is also reported that compared to normal or ACL patients, the RNA and protein expression levels demonstrated statistical differences in OA patients in samples such as synovial fluid, synovium and peripheral blood [25]. The different expression genes are supposed to contribute to the initiation and $\mathrm{OA}$ and have aroused universal concern, which are optical potential biomarkers of joint diseases. For instance, a report suggests that relevant biomarkers of joint disease are identified by metabolomic profiling of synovial fluid [26].

Then the differently expressed proteins in different groups were further analyzed (Fig.3). Although the proteins that might be changed due to aging, menopause and OA were identified, the pathways via which they might contribute to the joint function remaining or degeneration were still unclear. Protein-protein interaction might provide important clues in studying the molecular mechanisms that might be involved.

The reactions and pathways among immune or endocrine-related proteins which varied significantly in old OA women compared to young OA women were analyzed and visualized by the Reactome program (Fig.4), which might provide insight into the molecular mechanism of aging contributing to the development of OA.

\section{Conclusions}

In the study, protein expression profiles of synovial tissues from female knee OA and knee injury patients were analyzed. Around 4000 proteins were identified; 3465 proteins were analyzed. Our results revealed that protein expressions in ST were affected by both aging and menopause. Similarly, aging and menopause also influenced the post-surgery joint functional outcomes of these patients. Therefore, aging and menopause might affect the development of OA as well as the post-surgery recovery of the knee joint through changing the protein expression patterns of ST, we supposed. However, the exact effect of the protein components in ST on the occurrence, development, and rehabilitation of OA remains unclear. More studies are needed to further elucidate the influences of aging and menopause on the knee joint and throw light on the mechanism through which ST might affect the functions of the knee joint.

\section{Abbreviations}

\begin{tabular}{ll} 
GO & Gene Ontology \\
\hline KEGG & Kyoto Encyclopedia of Genes and Genomes \\
\hline OA & Osteoarthritis \\
\hline ST & Synovial Tissue \\
\hline WOMAC & The Western Ontario and McMaster Universities Osteoarthritis Index \\
\hline VAS & Visual Analogue Score
\end{tabular}

Page 5/12 


\section{Declarations}

\section{Acknowledgements}

The authors would like to thank the participants for their contribution and cooperation and Jiguang Gene Biotechnology Co.Ltd (Nanjing) for their help in proteome analysis.

\section{Authors' contributions}

X Yang and Z Fu collected the samples and follow-up data, QY Ren analyzed and interpreted the data, and wrote the manuscript. S Wang wrote the manuscript. QY Ren and S Wang collected clinical samples. S Yin and J Liu designed the study, analyzed the data and revised the manuscript. All authors read and approved the final manuscript.

\section{Funding}

This work was funded by Science \& Technology Department of Sichuan Province (NO. 2019YFS0318); Foundation of Tianjin Health Commission (ZC20192); These fundings are used for data collection, hormone levels detection, proteome analysis and interpretation of data.

\section{Availability of data and materials}

The datasets used and/or analyzed during the current study are available from the corresponding author on reasonable request.

\section{Ethics approval and consent to participate}

The study was approved by the ethics committee of Sichuan Academy of Medical Sciences $₫$ Sichuan Provincial People's Hospital.

\section{Consent for publication}

Not applicable.

\section{Competing interests}

The authors declare that they have no competing interests

\section{Author details}

1 Graduate School of Tianjin Medical University, Tianjin, China

2 Department of Orthopaedics, Sichuan Academy of Medical Sciences $₫$ Sichuan Provincial People's Hospital, Chengdu, China.

3 Department of Orthopaedics, Tianjin Hospital, Tianjin University, Tianjin, China

4 Department of Orthopaedics, Cangzhou Hospital of Integrated TCM-WM Hebei Province, China

\section{References}

1. Collins JA, Diekman BO, Loeser RF. Targeting aging for disease modification in osteoarthritis. Curr Opin Rheumatol. 2018; 30(1): 101-7. doi: 10.1097/BOR.0000000000000456

2. Sacitharan PK. Ageing and Osteoarthritis. Subcell Biochem. 2019; 91: 123-59. doi: 10.1007/978-981-13-3681-2_6

3. Vina ER, Kwoh CK. Epidemiology of osteoarthritis: literature update. Curr Opin Rheumatol. 2018; 30(2): 160-7. doi: 10.1097/BOR.0000000000000479

4. Lee $\mathrm{YH}$. Investigating the possible causal association of coffee consumption with osteoarthritis risk using a Mendelian randomization analysis. Clin Rheumatol. 2018; 37(11): 3133-9. doi: 10.1007/s10067-018-4252-6

5. Morales-Ivorra I, Romera-Baures M, Roman-Vinas B, Serra-Majem L. Osteoarthritis and the Mediterranean Diet: A Systematic Review. Nutrients. 2018; 10(8) pii: E1030. doi: 10.3390/nu10081030

6. Mahajan A, Patni R. Menopause and Osteoarthritis: Any Association ? J Midlife Health. 2018; 9(4): 171-2. doi: 10.4103/jmh.JMH_157_18

7. Ritter, Susan Y et al. "Proteomic analysis of synovial fluid from the osteoarthritic knee: comparison with transcriptome analyses of joint tissues." Arthritis and rheumatismvol. 65,4 (2013): 981-92. doi:10.1002/art.37823

8. Tang MW, Reedquist KA, Garcia S, Fernandez BM, Codullo V, Vieira-Sousa E et al. The prolactin receptor is expressed in rheumatoid arthritis and psoriatic arthritis synovial tissue and contributes to macrophage activation. Rheumatology. 2016; 55(12): 2248-59. doi: 
10.1093/rheumatology/kew316

9. Chang, Xiaotian et al. "Identification of proteins with increased expression in rheumatoid arthritis synovial tissues." The Journal of rheumatologyvol. 36,5 (2009): 872-80. doi:10.3899/jrheum.080939

10. Hsueh, Ming-Feng et al. "Biomarkers and proteomic analysis of osteoarthritis." Matrix biology : journal of the International Society for Matrix Biologyvol. 39 (2014): 56-66. doi:10.1016/j.matbio.2014.08.012

11. Liu W, Cui Z, Wang Y, Zhu X, Fan J, Bao G, Qiu J, Xu D. Elevated expression of beta1,4-galactosyltransferase-I in cartilage and synovial tissue of patients with osteoarthritis. Inflammation. 2012; 35(2): 647-55. doi: 10.1007/s10753-011-9357-x

12. Xie C, Mao X, Huang J, Ding Y, Wu J, Dong S, Kong L, Gao G, Li CY, Wei L. KOBAS 2.0: a web server for annotation and identification of enriched pathways and diseases. Nucleic Acids Res. 2011; 39(Web Server issue): W316-22. doi: 10.1093/nar/gkr483

13. Felson DT, Naimark A, Anderson J, KazisL, Castelli W, Meenan, RF. The prevalence of knee osteoarthritis in the elderly. The framingham osteoarthritis study. Arthritis Rheumatol. 2014;30(8): 914-8. doi: 10.1002/art.1780300811

14. Gobezie R, Kho A, Bryan Krastins. High abundance synovial fluid proteome: distinct profiles in health and osteoarthritis. Arthritis Res Ther. 2007; 9(2). doi: 10.1186/ar2172

15. Pan X, Huang L, Chen J, Dai Y, Chen X. Analysis of synovial fluid in knee joint of osteoarthritis:5 proteome patterns of joint inflammation based on matrix-assisted laser desorption/ionization time-of-flight mass spectrometry. Int Orthop. 2012;36(1), 57-64. doi: 10.1007/s00264011-1258-y

16. David S. Gibson, Madeleine E. Rooney. The human synovial fluid proteome: A key factor in the pathology of joint disease. Proteom Clin Appl. 2010; 1(8):889-99. doi: 10.1002/prca.200700044

17. Driban, Jeffrey B et al. "The potential of multiple synovial-fluid protein-concentration analyses in the assessment of knee osteoarthritis." Journal of sport rehabilitationvol. 19,4 (2010): 411-21. doi:10.1123/jsr.19.4.411

18. Ritter, Susan $Y$ et al. "Mass spectrometry assays of plasma biomarkers to predict radiographic progression of knee osteoarthritis." Arthritis research \& therapyol. 16,5 456. 7 Oct. 2014, doi:10.1186/s13075-014-0456-6

19. Luo Q , Ji S , Li Z , Luo Q, Ji S, Li Z, Huang T, Fan S, Xi Q. Effects of ultrasound therapy on the synovial fluid proteome in a rabbit surgeryinduced model of knee osteoarthritis. Biomed Eng Online. 2019; 18(1). doi: 10.1186/s12938-019-0637-2

20. Svala E, Jin C, Rüetschi U, Ekman S, Lindahl A, Karlsson NG, Skiöldebrand E. Characterisation of lubricin in synovial fluid from horses with osteoarthritis. Equine Vet J. 2017;49(1):116-23. doi: 10.1111/evj.12521

21. Roman-Blas JA, Castañeda S, Largo R, Herrero-Beaumont G. Osteoarthritis associated with estrogen deficiency. Arthritis Res Ther. 2009;11(5):241. doi: 10.1186/ar2791.

22. Martín-Millán M, Castañeda S. Estrogens, osteoarthritis and inflammation. Joint Bone Spine. 2013;80(4):368-73. doi: 10.1016/j.jbspin.2012.11.008.

23. Spector TD, Nandra D, Hart DJ, Doyle DV. Is hormone replacement therapy protective for hand and knee osteoarthritis in women? The Chingford Study. Ann Rheum Dis. 1997;56:432-44. doi: 10.1136/ard.56.7.432.

24. Zhang Y, McAlindon TE, Hannan MT, Chaisson CE, Klein R, Wilson PW, Felson DT. Estrogen replacement therapy and worsening of radiographic knee osteoarthritis: the Framingham Study. Arthritis Rheum. 1998;41:1867-73. doi: 10.1002/15290131(199810)41:10<1867::AID-ART20>3.0.C0;2-W.

25. Dang X, Lian L, Wu D. The diagnostic value and pathogenetic role of IncRNA-ATB in patients with osteoarthritis. Cell Mol Biol Lett. 2018; 23: 55. doi: 10.1186/s11658-018-0118-9

26. Carlson AK, Rawle RA, Adams E, Greenwood MC, Bothner B, June RK. Application of global metabolomic profiling of synovial fluid for osteoarthritis biomarkers. Biochem Bioph Res Co. 2018; 499(2): 182-8. doi: 10.101

\section{Tables}

\section{Table 1 Patients characteristics}




\begin{tabular}{|llll|}
\hline Variable & Postmenopausal OA women & Premenopausal OA women & Premenopausal knee injury women \\
\hline Number $(\mathrm{n})$ & 3 & 3 & 2 \\
\hline Mean age $(\mathrm{M} \pm \mathrm{SD}, \mathrm{y})$ & $63.3 \pm 5.8$ & $34.7 \pm 5.7$ & $40.5 \pm 0.7$ \\
\hline Affected knee (left/right, $\mathrm{n})$ & $2 / 1$ & $1 / 2$ & $2 / 0$ \\
\hline BMl & $24.7 \pm 1.2$ & $21.3 \pm 1.1$ & $20.6 \pm 0.3$ \\
\hline Estrogen $(\mathrm{pg} / \mathrm{mL})$ & $<11.8^{*}$ & $138.9 \pm 48.4$ & $101.1 \pm 35.9$ \\
\hline Evaluated scores $(\mathrm{pre} /$ one-month post-surgery) & & $6.5 \pm 0.7 / 1.0 \pm 0.1$ \\
\hline VAS & $6.7 \pm 0.58 / 2.0 \pm 1.0$ & $6.3 \pm 0.6 / 1.3 \pm 0.6$ & $67.0 \pm 1.4 / 90.5 \pm 2.1$ \\
\hline Lysholm & $69.3 \pm 1.16 / 81.3 \pm 4.0$ & $65.3 \pm 5.0 / 92.3 \pm 2.1$ & $144.0 \pm 5.7 / 69.0 \pm 1.4$ \\
\hline WOMAC & $148.7 \pm 7.02 / 106.3 \pm 10.0$ & $116.7 \pm 20.8 / 71.0 \pm 3.6$ & \\
\hline
\end{tabular}

BMI: body mass index; VAS: the visual analog scale; WOMAC: the Western Ontario and Mcmaster Universities Osteoarthritis Index.

*Estrogen levels of postmenopausal women are all less than 11.8.

Table 2 The top 20 proteins varied greatly in different groups

\begin{tabular}{|c|c|c|c|c|c|c|c|c|}
\hline \multicolumn{3}{|c|}{ young vs old } & \multicolumn{3}{|c|}{ post-vs pre-menopausal } & \multicolumn{3}{|c|}{ OA vs knee injury } \\
\hline Accession & GeneSymbol & $\log 2(Y / O)$ & Accession & GeneSymbol & Log2(post/pre) & Accession & GeneSymbol & Log2(OA/injury) \\
\hline 043488 & AKR7A2 & 0.4269663 & Q9BTE1 & DCTN5 & 0.289498664 & Q9NXG2 & THUMPD1 & 0.269383267 \\
\hline Q9GZT8 & NIF3L1 & 0.36240516 & 043488 & AKR7A2 & 0.409239429 & Q96T23 & RSF1 & 0.277527593 \\
\hline P30876 & POLR2B & 0.26354796 & Q9NX24 & NHP2 & 0.302979617 & Q9ULT0 & TTC7A & 0.289193504 \\
\hline P07333 & CSF1R & 0.493512404 & 000160 & MY01F & 0.275863833 & Q9Y5P4 & COL4A3BP & 0.290021778 \\
\hline Q9Y2S2 & CRYL1 & 0.477158749 & P52630 & STAT2 & 0.294097071 & P63172 & DYNLT1 & 0.296420131 \\
\hline Q658P3 & STEAP3 & 0.386304842 & Q15257 & PTPA & 0.283669719 & Q9UKK9 & NUDT5 & 0.316220545 \\
\hline Q5SRE7 & PHYHD1 & 0.496614233 & P07333 & CSF1R & 0.443399472 & 095858 & TSPAN15 & 0.348805567 \\
\hline P48426 & PIP4K2A & 0.361906826 & 014773 & TPP1 & 0.31591994 & Q9NX14 & NDUFB11 & 0.395980419 \\
\hline Q15006 & EMC2 & 0.577473851 & Q15006 & EMC2 & 0.682535623 & Q13523 & PRPF4B & 0.521081568 \\
\hline Q9BRG1 & VPS25 & 0.380148739 & Q9Y2S2 & CRYL1 & 0.399596671 & P07947 & YES1 & 0.6417041 \\
\hline Q00013 & MPP1 & 0.336292433 & P55039 & DRG2 & 0.373345031 & P41219 & PRPH & 0.906690157 \\
\hline Q96G23 & CERS2 & 0.602784193 & Q9Y3E1 & HDGFL3 & -0.298723752 & Q9Y570 & PPME1 & -0.502189867 \\
\hline Q01844 & EWSR1 & 0.356185854 & 075592 & MYCBP2 & -0.281459426 & 095219 & SNX4 & -0.440872985 \\
\hline 000487 & PSMD14 & -0.3046601 & P62841 & RPS15 & -0.371475399 & Q15370 & ELOB & -0.360115548 \\
\hline 095182 & NDUFA7 & -0.39374881 & Q9UMS6 & SYNPO2 & -0.441555468 & Q13232 & NME3 & -0.342458488 \\
\hline P53992 & SEC24C & 0.267462867 & Q2TAA2 & $\mathrm{IAH} 1$ & -0.309676687 & Q15427 & SF3B4 & -0.274833517 \\
\hline Q9UK45 & LSM7 & 0.314089197 & Q99729 & HNRNPAB & -0.325274373 & Q13445 & TMED1 & -0.273530095 \\
\hline Q9NZD2 & GLTP & 0.335007761 & Q13620 & CUL4B & -0.381895597 & P56545 & СТВP2 & -0.47964211 \\
\hline P05388 & RPLPO & 0.286252632 & P14621 & ACYP2 & -0.329073087 & Q14938 & NFIX & -0.435821005 \\
\hline P61513 & RPL37A & -0.60955358 & 014495 & PLPP3 & -0.385721448 & Q15628 & TRADD & -0.362026664 \\
\hline
\end{tabular}


Figures

Fig.1

a
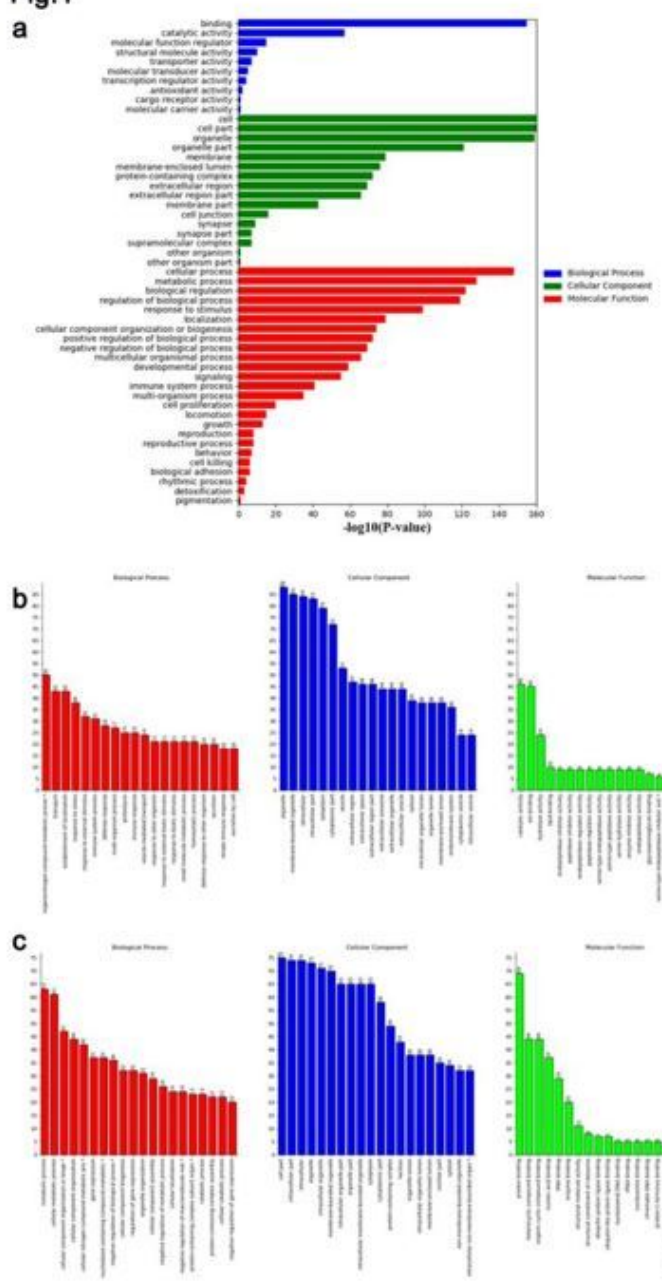
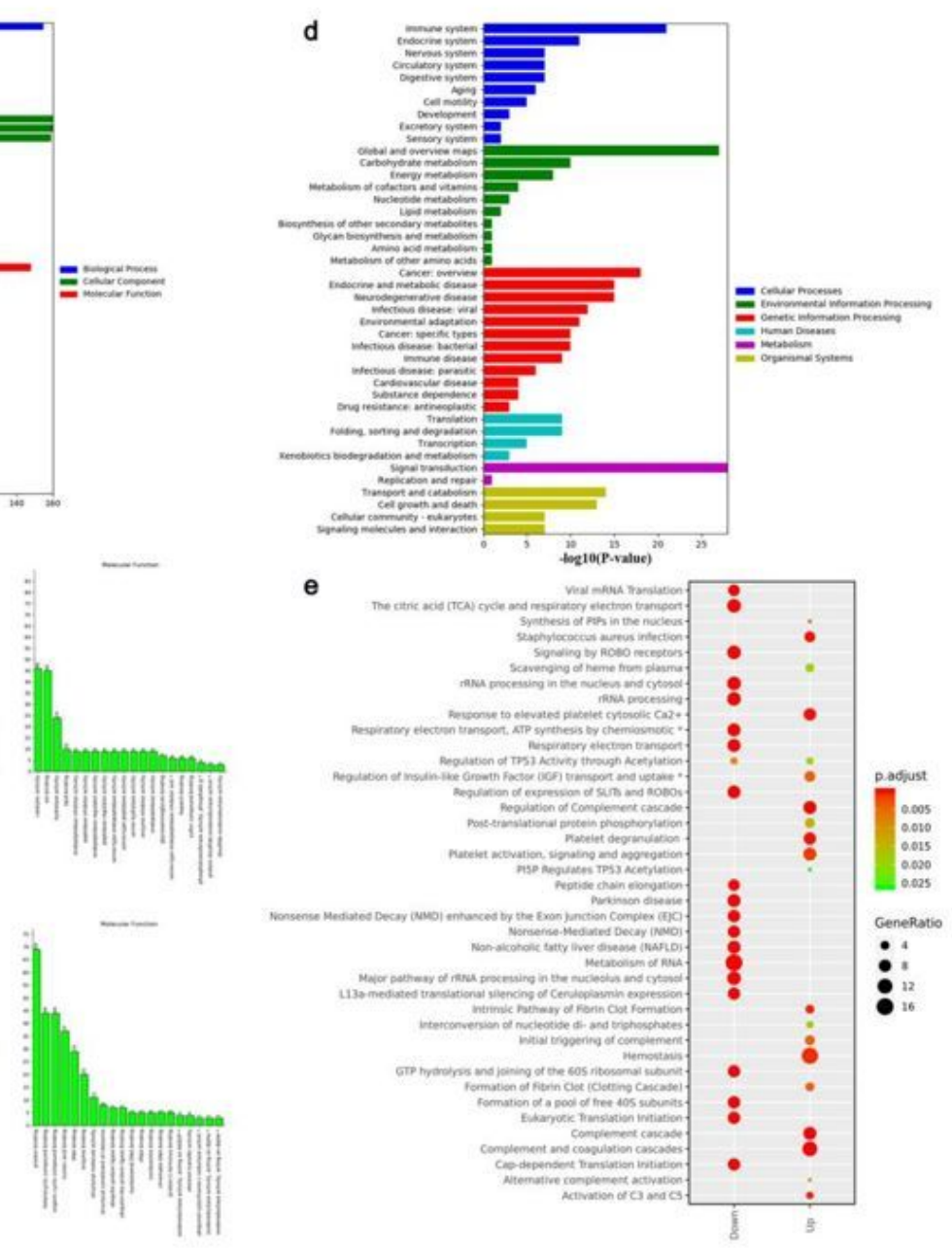

\section{Figure 1}

GO and KEGG analysis of synovial tissues (ST) from knee osteoarthritis (OA) and injury women. a. GO analysis of the biological functions of proteins in ST. b-c The top 20 proteins increased (b) vs decreased (c) in ST from young women ( $<45 \mathrm{y})$ compared to the old ones (>60y). $d$ The biological functions of the detected proteins analyzed by KEGG. e and involved biological processes of the detected proteins analyzed by KEGG. 

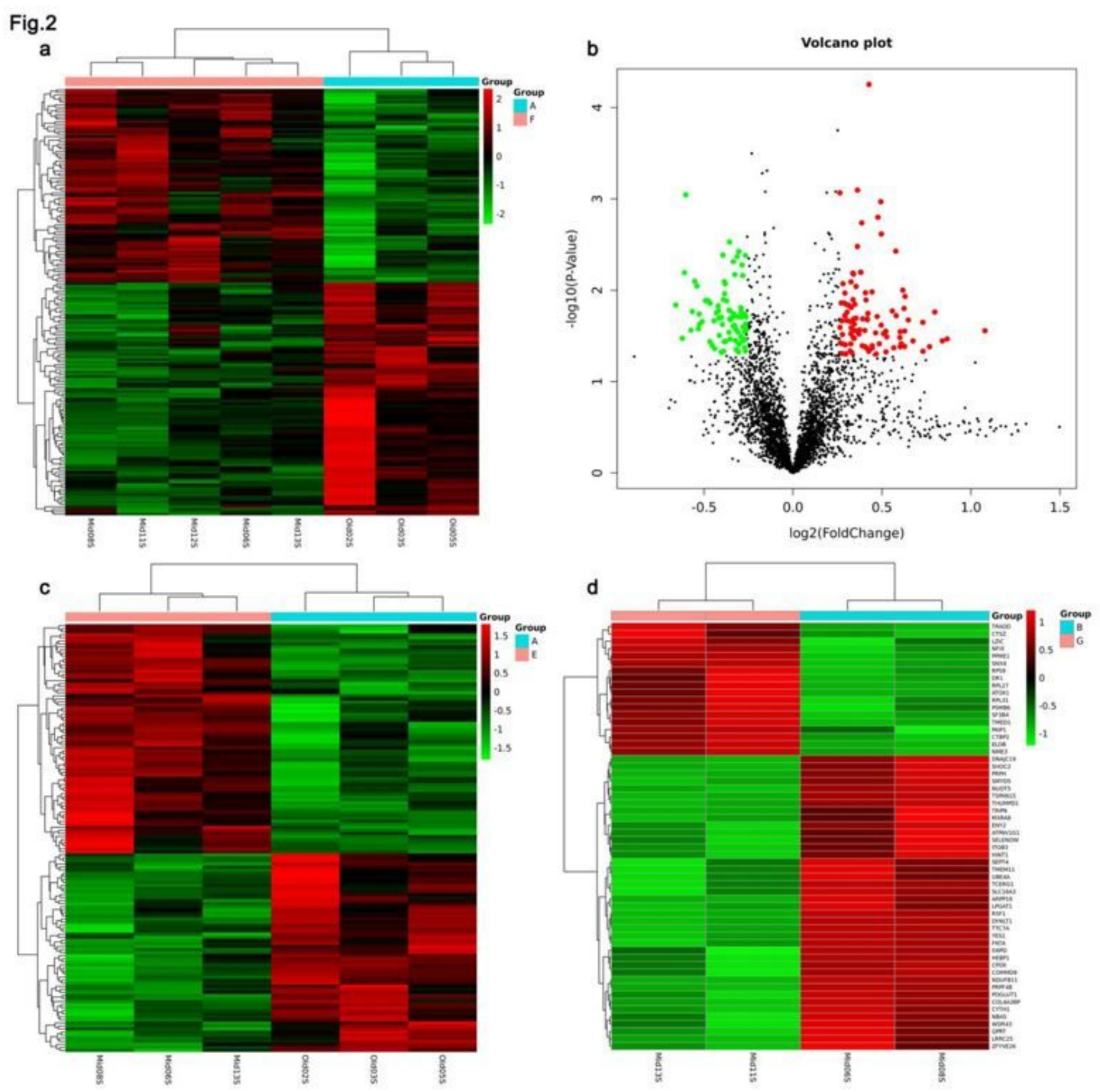

\section{Figure 2}

The differently expressed proteins in synovial tissues (ST) samples from different groups. a-b The heatmap (a) and the volcano plots (b) of the differently expressed proteins between aging and young knee osteoarthritis (OA) women. c The heatmap of ST proteins from postmenopausal and menopausal OA women. $d$ The heatmap of ST proteins between menopausal knee OA women and menopausal knee injury women. 
Fig.3
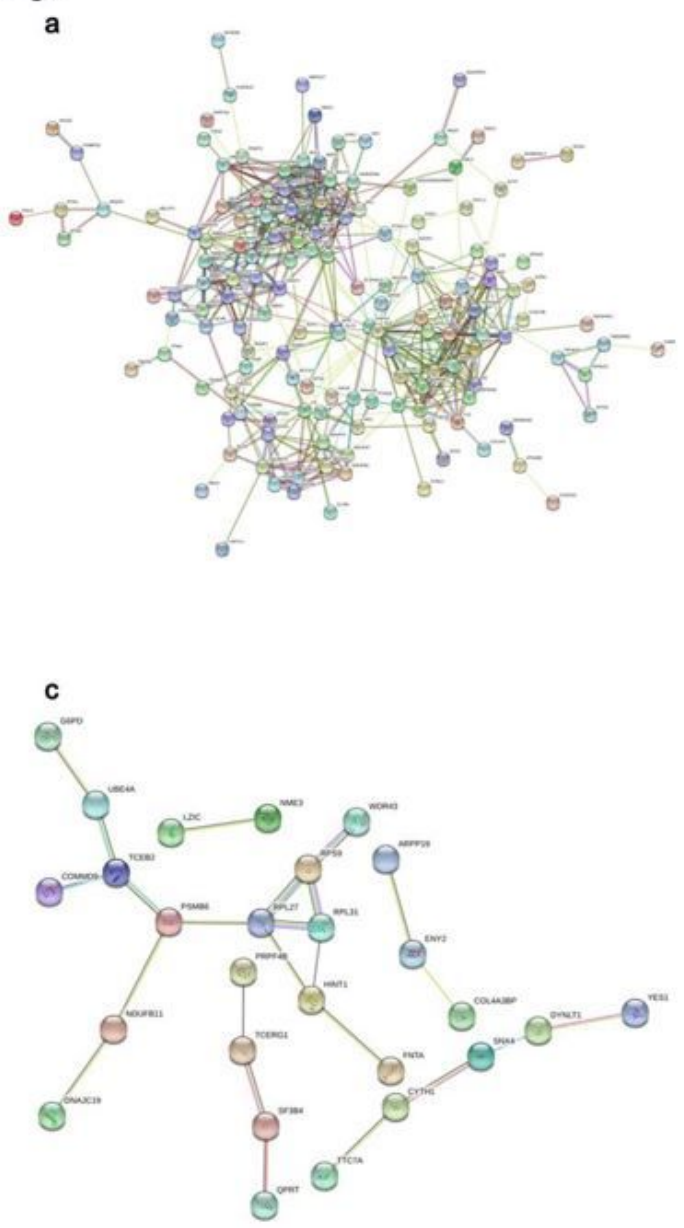
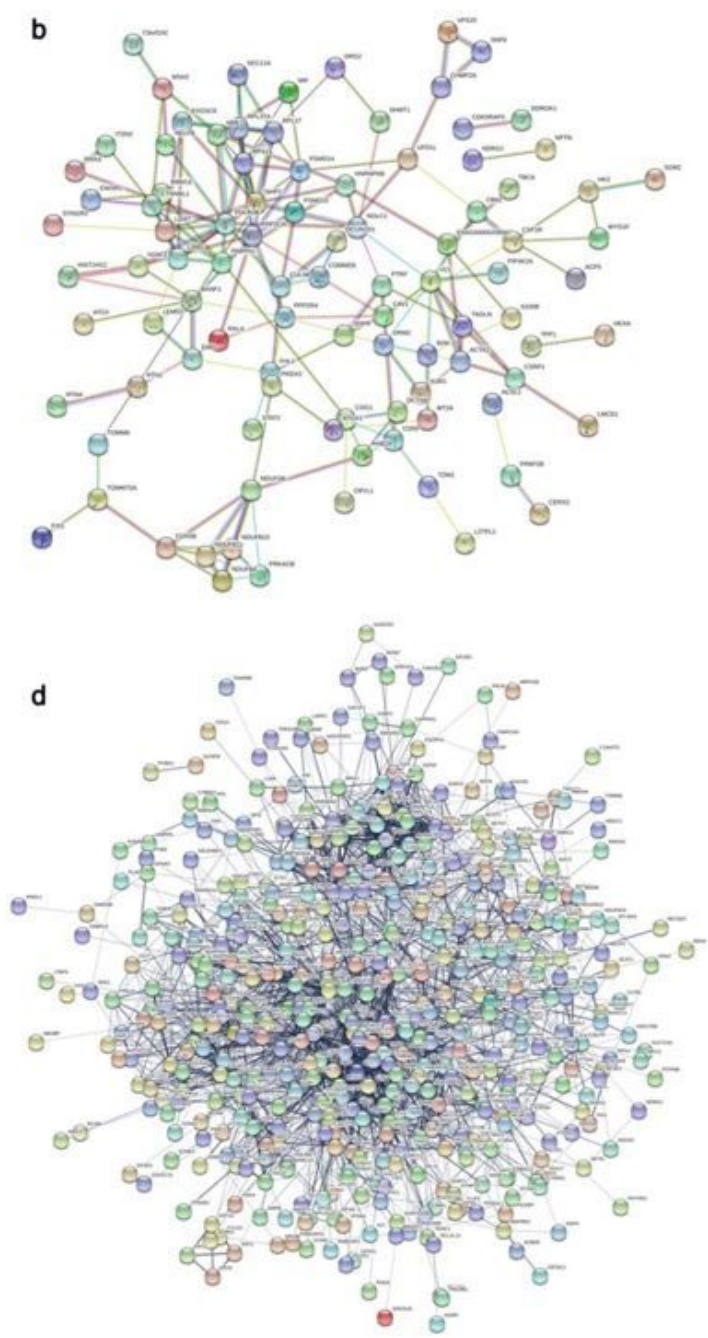

\section{Figure 3}

The protein-protein interaction network analyzed by STRING. a The protein-protein interaction network of the proteins most affected by aging from synovial tissues (ST) of knee osteoarthritis (OA) women. b The protein-protein interaction network of the proteins varied greatly with menopause in synovial tissues (ST) of knee osteoarthritis (OA) women. c The protein-protein interaction network of the proteins statistical different between knee OA and injury women. $d$ The protein-protein interaction network of the proteins statistical different in ST samples from OA women with high and low estrogen level.

\section{Fig.4}

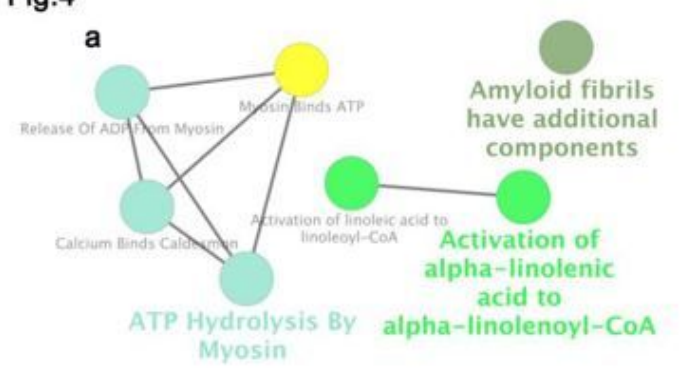

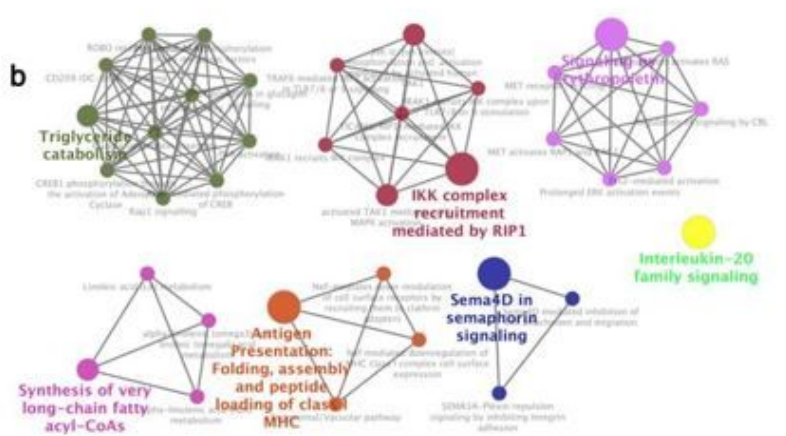

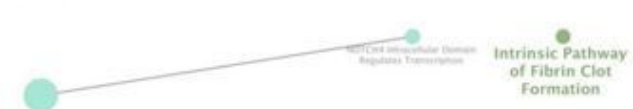


Figure 4

The reactions and pathways analyzed by Reactome. The reactions (a) and the pathways (b) among the immune or endocrine-related proteins varied significantly in synovial tissues (ST) between menopausal and premenopausal osteoarthritis (OA) women.

\section{Supplementary Files}

This is a list of supplementary files associated with this preprint. Click to download.

- SupplementaryMaterial.rar 